de Navigation and Deutsche Gesellschaft für Ortung und Navigation, agreed to set up a working group to determine and propose solutions for the areas in which the problem is most acute. The working group would comprise the representatives of shipping interests as well as experts from the various organizations which would have the technical responsibility for establishing the routes. In addition to making recommendations for the separation of traffic the group would consider the navigational aids which might be required.

Concerning air travel the discussion on supersonic transport indicated that a deeper investigation of the environment and its influence on the specification of the navigation system was necessary. The conference agreed that here the Institutes could perhaps make a unique contribution by clarifying the governing parameters and co-ordinating the various investigations that had so far been made. The three Institutes agreed to form an international working group to investigate the whole problem.

Further information concerning the conference can be obtained from Mr. M. W. Richey, executive secretary, the Institute of Navigation, at the Royal Geographical Society, 1 Kensington Gore, London, S.W.7.

\title{
THE DIELECTRICS EVAPORATION GROUP
}

$\mathrm{T}$ HE Dielectrics Evaporation Group was formed some five years ago under tho auspices of the Ministry of Aviation to promote the interchange of information between active workers in the field of evaporated thin dieleatric films and relevant subjects such as formation and properties of the metal electrodes.

Meetings are held every six months at some centre of research at which a number of informal papers dealing with the most recent research topics are delivered. These are reported in the form of notes on the meetings which are duplicated and distributed to all scientists with a genuine interest in the subject. Attendance at the meetings is by invitation and is restricted to active workers capable of making a significant contribution.

The Group is now under the patronage of the Joint British Council for Vacuum Studies and under the chairmanship of Mr. L. Holland (Edwards High Vacuum, Ltd.).

The tenth meeting of the Dielectrics Evaporation Group was held on March 25 at the Plessey Research Laboratories, Caswell. The following papers were read: "A Survey of the Resistive and Conductive Properties of Thin Metal Films", by Mr. J. Bennett (British Scientific Instrument Research Association); "The Mechanism of Conduction in Thin Films", by Dr. R. Hill (Electrical Research Association); "Structure Studies on NickelChromium Films", by Mr. H. Blackburn (Plessey Research Laboratories, Caswell); "The Quartz Crystal Rate-Meter", by Mr. H. Bath (Edwards Hi-Vacuum); "Studies of Rate Controlled Growth inside the Electron Microscope", by Messrs. D. J. Stirland, D. Carpenter and D. Dobbs (Plessey Research Laboratories, Caswell); "Studies of Thin Film Active Devices", by Mr. J. Acton (Plessey Research Laboratories, Caswell); "Vapour Phase Deposition of Thin Films", by Mr. B. A. Joyce (Plessey Research Laboratories, Caswell); "Deposition of Germanium Films", by Dr. J. C. Courvoisier (Battelle Research Institute, Geneva); "Differences in Growth Characteristics on Air and Vacuum Cleaved Substrate Surfaces", by Dr. O. S. Heavens (Royal Holloway College); "The Scanning Electron Microscope as a Means of Surface Study", by Mr. A. N. Broers (Department of Electrical Engineering, University of Cambridge).

From these papers some points emerged as worthy of special notice.

The conduction processes in very thin metal films are not yet fully understood. Such films usually consist of incompletely aggregated island structures on an insulating substrate. Conduction must therefore consist of the passage of electrons from island to island either directly or by means of the substrate. Dr. Hill considered that the former process may be discounted as leading to unacceptable values of resistance and temperature coefficient. He preferred the mechanism based on the passage of electrons to unoccupied levels in the macroscopically amorphous substrate and the ejection of equivalent electrons from the substrate into other such metal islands at a lower potential. Such a view could be consistent with Fröhlich's ideas on the existence of localized band structures in glassy materials. The small energy gaps would be overcome by the effects of the space charge developed. Results obtained by rough calculation were in reasonable agreement with experimental results for gold films on glass substrates, for which the island structure has been determined by electron microseopy.

Such an island structure had been observed by $\mathbf{M r}$. Blackburn, who had been studying the stability of evaporated nickel-chromium alloy resistors. It consisted of metal islands embedded in a glassy medium thought to be chromic oxide. Dr. Hill's proposed conduction mechanism could here well apply.

The processes of nucleation and growth (leading eventually to the island structure) depend critically on substrate conditions and residual atmosphere. Dr. Heavens showed that single crystal substrates freshly cleaved give very different results according to whether this has been done in air or in a good vacuum. The characteristic cleavage stop decoration observed for air cleaved substrates gave way to a random distribution of nucleation sites. The coalescence of these nuclei gave evidence of a high degree of orientation at much lower temperatures than is ordinarily the case. These effects were observed when the rate of arrival of metal atoms was at least comparable with that of the residual gas molecules (pressure approximately $2 \times 10^{-7} \mathrm{~mm}$ mercury).

Similar effects had been observed in the preparation of epitactic films of germanium by M. Courvoisier, using a poor vacuum, but depositing at a very high rate on a substrate heated almost to its melting point.

Nucleation processes very similar to those observed in vacuum deposition were described by Mr. Joyce for the vapour phase deposition of silicon from organic compounds.

The scanning electron microscope, with its large depth of focus and small electron spot size $(\sim 100 \AA)$, is emerging as a powerful tool for the study of surfaces. Mr. Broers described its use in combination with controlled ionbombardment etching for investigating such structures as thermally grown oxide films on iron, to which normal electron microscopy could not readily be applied. The depth of progressive etching of the surface can be controlled $( \pm 100 \AA)$ so that examination of the various layers can then be carried out with the electron probe. (Secondary emission during the ion bombardment makes it necessary to interrupt the etching during the electron scan.) The possible applications of this technique to thin films are clear. Such a process can be applied to the investigations of $p$ or $n$ conductivity in semiconductors, which emit secondary electrons of slightly different energies, although resolution may not be so high.

It is hoped the next meeting of the Group will be held some time in October.

J. R. BALMER

J. H. Brude 